\title{
Hazard sign comprehension among illiterate adults
}

\author{
Amanda Nicol and Seppo Tuomi \\ Department of Speech-Language and Hearing Therapy, Faculty of Health Sciences, \\ Stellenbosch University, P.O.Box 19063, 7505 Tygerberg \\ Email: stuomi@sun.ac.za
}

\section{Introduction}

Hazard signs have been considered an effective mode of transferring safety information for many decades. However, research is beginning to show that many of these signs are not understood, resulting in increasing numbers of preventable accidents (Wilkinson, Cary, Barr, and Reynolds 1997; Department of Trade and Industry (DTI) 1999; Occupational and Environmental Health Research Unit (OEHRU) 2003; Kalaroa 2005; Lesch 2005). This is particularly the case in South Africa, where poisoning and burn victims are seen daily in our hospitals. In the review below, factors that have been found to influence sign comprehension are discussed. This is followed by an overview of research pertaining to people's understanding of hazard signs. The chapter concludes by proposing two research questions aimed at evaluating how illiterate adults in South Africa comprehend selected hazard signs.

To explore the intricate world of sign comprehensibility it is necessary to establish an understanding of the field of semiotics. Semiotics in its broadest sense "comprises all forms of formation and exchange of meaning on the basis of phenomena which have been encoded as signs" (Johansen and Larsen 2002: 3). In layman's terms, semiotics is the study of signs, their meanings, interactions and interpretations (Andrews 2000). It thus serves as an effective framework for investigating signs. 
According to C.S. Peirce, the founder of modern semiotics, a sign consists of three interconnected parts (Kristeva 1989; Chandler,1994; Andrews 2000; Johansen and Larsen 2002):

1. The representamen or sign proper. This includes a sign's inner ground, internal structure, character and quality. The representamen is that which represents something else.

2. The object. This is the correlate for which the sign stands. It is that which is represented by the sign.

3. The interpretant or potential meaning that the sign allows.

It goes beyond the scope of this discussion to investigate these three elements in greater detail. Suffice to say that their interaction is essential for the comprehension of all types of signs.

At this point it is useful to explore iconicity, which is another important concept within the field of semiotics. "Iconicity" is a general term referring to the degree of visual similarity between a sign and its referent (Dunham 1989; Soto and Olmstead 1993; Blischak, Lloyd and Fuller 1997; Andrews 2000). Mizuko (2004) describes iconicity as a continuum that categorizes signs by ease of recognition. At either end of this continuum are transparent signs and opaque signs, respectively.

Transparent signs visually resemble their referents and, therefore, have highly guessable meanings. Hollosi (2000) notes that the meanings of such signs are more or less independent of the person interpreting them and can therefore be useful in international communication. However, Hollosi (2000) also notes important limitations to this independence in interpretation. Firstly, highly transparent signs can only represent real objects, thereby excluding abstract concepts such as freedom, love, anger, sickness, and warning. Secondly, perceptions of these signs are culturally dependent. This may result in signs assuming different meanings (or losing their meaning) in different cultures. These factors are discussed in more detail further on. 
Opaque signs, on the other hand, do not have an obvious relationship to their referents and may be quite arbitrary. Such signs are not limited to real objects and can represent a variety of complex meanings and ideas (Hollosi 2000). For this reason they may serve as an effective means of communication. However, these signs have an important limitation because they are not immediately recognizable. Their efficacy for communication requires that their meanings are first learned.

For the purposes of the present study, iconicity is an essential variable to consider when selecting specific hazard signs, because signs with a high degree of iconicity (that is, highly transparent signs) are more likely to be instantly recognizable and therefore preferable for use (Fuller and Lloyd 1997; Hollosi 2000; Johansen and Larsen 2002). This is a particularly important factor in countries with high levels of illiteracy, such as South Africa (Basson and Alant 2005). Iconicity has also been found to be an important factor determining the ease with which signs and their meanings are learnt and remembered (Fristoe and Lloyd 1979; Fuller and Stratton 1991; Fuller and Lloyd 1997).

The question, however, remains: What influences an individual's ability to understand signs? The answer can be found in the description of two groups of variables, namely picture variables and individual variables. Picture variables are factors found within the signs themselves. According to Johansen and Larsen (2002), signs are made up of elements which are determined by two or more properties, such as form and colour, found in all pictograms and shown to influence the ease with which pictograms are both interpreted and remembered (Kristeva 1989; Hanna and Remington 1996; Johansen and Larsen 2002; Wilkinson and Jagaroo 2004). Other researchers also include shape, size, location/context, material, openness and intelligibility as factors that may influence sign perception, interpretation and recall (Terumasa and Takeshi 1999; Tsal and Bareket 1999; Davies, Haines, and Norris 2000; Mizuko 2004). A major advantage of these variables is their adaptability for use in different contexts.

Individual variables are found within the individual. Unlike picture variables, individual variables may not be easy to modify. They are, therefore, vital to consider in the context of hazard sign comprehension, since an inherent failure to perceive, interpret and remember such signs may lead to serious injury and even death. Researchers have identified a variety of 
individual factors that are of relevance (cf. Stephenson and Linfoot 1996; Huer 2000; Wilkinson and Jagaroo 2004; Kalaroa 2005; Lesch 2005). Factors specifically pertaining to the South African context, namely education, literacy, language, culture, and previous experience, will be discussed below.

Problems due to inadequate education and literacy in South Africa have been well documented (van Heerden 1991; Underwood 2002). Although the available statistics are not completely reliable, adult illiteracy figures conservatively stand at 15.1\% (United Nations Development Program 2002). In a study that contrasted illiteracy figures in urban and rural areas, adult illiteracy was found to be as high as $27 \%$ and $50 \%$ respectively (Ministry for Welfare and Population Development 1997). The discrepancy may be due to the fact that to be classified as a literate person in South Africa requires more than just the ability to read. According to the Workforce Investment Act (1998), to be literate includes the ability to read, write, talk, reason and solve problems, so that one can successfully function within the work environment, family and community. The inability to function at this level is termed "functional illiteracy", and has been estimated to be as high as 70\% (van Heerden 1991).

These low percentages result in two opposing implications for hazard sign comprehension. Firstly, the high prevalence of adult illiteracy should serve as a motivation for the use of pictorial hazard signs as helpful alternatives to written warnings. The iconicity of these signs is important to consider since those with higher degrees of transparency will be more easily recognizable and therefore more useful in an illiterate environment (Hollosi 2000; Johansen and Larsen 2002; Basson and Alant 2005). On the other hand, the high level of illiteracy does not bode well for the acquisition and use of pictorial signs, because both written words and pictorial signs are symbolic systems which rely on the use of a representamen to symbolize or represent something that is not present (Kristeva 1989; Johansen and Larsen 2002). A literate person, when shown an unknown pictorial sign, may be more easily able to generalize the knowledge of grapheme-concept relationships to form a picture-concept relationship, thereby aiding comprehension of the unknown sign.

The negative impact of illiteracy on hazard sign comprehension is exacerbated by the fact that South Africa has no standardized warning system to which all sectors adhere (Global Harmonized System (GHS) for the Classification and Labeling of Chemicals Study 2003). As 
a result, there is a wide variety of hazard signs in use with which many South Africans are not adequately acquainted. Since the consistency and prevalence of signs have been shown to directly affect their comprehension and recall (Johansen and Larsen 2002), hazard sign comprehension in South Africa is likely to be affected. The GHS (2003) study confirmed this by testing the comprehensibility of twelve hazard symbols that appear on industrial and household chemicals across the four economic sectors of industry, transport, agriculture, and consumers. Findings from over 400 participants revealed that only two symbols, namely the "skull-and-crossbones" symbol and the "flammable" symbol, had acceptable levels of comprehensibility, being understood by more than $50 \%$ of the participants. For six of the symbols tested, less than $50 \%$ of the participants were able to provide correct or even partially correct responses.

Interestingly, the GHS (2003) study revealed a difference in the comprehension of pictograms and hazard symbols. Advisory pictograms indicating the need for gloves, boots, hand washing after use, etc., elicited over 90\% correct or partly correct responses (GHS Study 2003). This finding, along with the finding that symbols are key to attracting attention to risk, forms the proverbial silver lining around the cloud of sign comprehensibility.

The findings of the GHS (2003) study concur with a number of similar studies in both the United Kingdom and the United States of America, indicating that hazard signs are generally poorly understood (DTI 1999; Kalaroa 2005; Lesch 2005). A variety of explanations have been suggested for this, including low levels of public education and literacy, and a lack of hazard sign training.

Another likely factor in the lack of understanding of hazard signs is the role of multicultural and multilingual contexts. South Africa has eleven official languages and many cultures inextricably linked to these languages (Underwood 2002). A study by Huer (2000), based in the USA, addressed the issue of cross-cultural differences in sign comprehension. This study examined the perception of three graphic symbol sets across 147 adults from four different cultural groups, namely African American, Chinese, European American, and Mexican. Significant differences between these cultural groups were found regarding the transparency ratings assigned to the symbols. Although these results may have limited generalizability within the South African context, it is conceivable that the vast number of languages and 
cultures in South Africa would result in similar findings. This has important implications for the design of hazard signs, as preference should be given to those that are as culturally unbiased as possible.

With only limited information on hazard sign comprehensibility available in the South African context, further research is sorely needed before suitable symbol selections can be effectively made. Sound theoretical research is needed which outlines the factors that influence sign comprehensibility within the South African context. It is with this in mind that the following two research questions were posed and investigated in the present study. Firstly, how effective are hazard signs in transmitting information to illiterate adults? This research question aimed to quantitatively assess the comprehensibility of eight common hazard signs amongst illiterate adults. Secondly, what is the most effective way in which these hazard signs should be presented? To answer this question, the comprehension of three different forms of the eight hazard signs was compared in order to determine the most easily understood form.

\section{Methodology}

\subsection{Research design}

An experimental group design using a sample survey format was selected for this study. Raw data were analyzed quantitatively. Some qualitative data regarding ways in which hazard signs could be improved was also collected. The data collection was done on a once-off basis, making the study cross-sectional in nature. Advantages of such a design include the use of randomization and a lack of maturation bias (Bless and Higson-Smith 2004).

\subsection{Aims}

The main aim of this study was two-fold. Firstly, the researchers wanted to determine how well the eight selected hazard signs were understood by illiterate adults in a low socioeconomic bracket. Secondly, the aim was to determine the most effective way in which these hazard signs should be presented to illiterate adults. These two aims were addressed by means of three processes:

1. Ranking of the eight hazard signs in order of comprehensibility ranging from the easiest to understand to the most difficult to understand. 
2. Comparison of the different types of hazard sign presentation to determine which was easiest to understand.

3. Eliciting suggestions from the participants for improvements in the design and presentation of hazard signs that could increase their comprehensibility.

\subsection{Participants}

\subsubsection{Participant Selection Criteria}

Due to the limited scope of the study, stringent selection criteria were instituted in order to control for as many within-participant variables as possible. All participants were required to be over the age of 25 years, and to speak Afrikaans as their first/home language. These criteria were established to eliminate the possible influence of language on results. Participants were also required to be illiterate. To ensure this, participants had to have enrolled in the Matie Community Service (MCS) Adult Basic Education and Training Programme within the past year, working towards their level 1 exam. Potential participants who had been enrolled in this programme longer than a year were excluded, as they might no longer be considered illiterate. For practical purposes, participants were also required to live in or around the Stellenbosch area, as this increased the likelihood of attendance at data gathering sessions. Lastly, only participants of low socio-economic status were included. Low socio-economic status was defined as a position of economic hardship or disadvantage, in which resources are inadequate and financial income is limited or non-existent (Barbarin and Richter 2001). The high prevalence of illiteracy in this population (cf. Underwood 2002), as well as the increased likelihood of contact with hazardous substances in many jobs held by individuals in this socio-economic bracket (OEHRU 2003), makes low socio-economic status an important criterion for this study. 


\subsubsection{Participant Selection Procedure}

Twenty-one individuals meeting the criteria were selected from those attending the adult education programme. They were randomly assigned to one of three groups consisting of seven participants each. Participants were contacted via the MCS Adult Basic Education and Training Programme after approval was obtained from the programme manager. A signed letter of consent was required from each participant. Due to the fact that participants were not able to read, the consent procedure as well as a careful outline of the study was verbally explained beforehand. Opportunities to ask questions were provided, and participants' rights within the research process were clearly explained.

\subsubsection{Participant Description}

All participants met the selection criteria stated above. The employed participants held jobs that fell into a low income category. All participants spoke Afrikaans as their home language and had been enrolled in the MCS Adult Basic Education and Training Program for no longer than a year. Information on mean age, gender distribution, and employment distribution for each group is provided in Table 1. The participant numbers in this table refer to the order in which participants were interviewed.

Two out of the 21 participants had not received formal schooling of any kind. These two were randomly placed in group 2 . The remaining participants had received between one and six years of schooling. No participants had completed their primary school education. Previous work experience with hazardous substances and equipment constituted the greatest differences between groups. These differences are also indicated in Table 1. 


\begin{tabular}{|c|c|c|c|c|c|c|}
\hline Group & $\begin{array}{l}\text { Participant } \\
\text { number }\end{array}$ & $\begin{array}{c}\text { Gender } \\
(\mathbf{M} / \mathbf{F})\end{array}$ & $\begin{array}{l}\text { Currently } \\
\text { employed }\end{array}$ & $\begin{array}{l}\text { Age } \\
\text { (yrs) }\end{array}$ & $\begin{array}{l}\text { Previous school } \\
\text { experience (yrs) }\end{array}$ & $\begin{array}{c}\text { Previous work } \\
\text { experience with (1) } \\
\text { chemicals, (2) electrical } \\
\text { equipment, (3) heavy } \\
\text { machinery }\end{array}$ \\
\hline 司 & $\begin{array}{c}1 \\
5 \\
10 \\
12 \\
15 \\
16 \\
21 \\
M\end{array}$ & $\begin{array}{c}\mathrm{M} \\
\mathrm{M} \\
\mathrm{M} \\
\mathrm{M} \\
\mathrm{F} \\
\mathrm{F} \\
\mathrm{M}\end{array}$ & $\begin{array}{l}\text { yes } \\
\text { yes } \\
\text { yes } \\
\text { yes } \\
\text { yes } \\
\text { yes } \\
\text { yes }\end{array}$ & $\begin{array}{r}52 \\
43 \\
48 \\
63 \\
46 \\
32 \\
63 \\
\mathbf{4 9 . 5 7}\end{array}$ & $\begin{array}{l}4 \\
4 \\
5 \\
5 \\
3 \\
6 \\
1 \\
4\end{array}$ & $\begin{array}{c}1,2,3 \\
3 \\
1,3 \\
1 \\
\text { No experience } \\
\text { No experience } \\
1,2\end{array}$ \\
\hline 䏠 & $\begin{array}{c}2 \\
3 \\
7 \\
9 \\
14 \\
18 \\
19 \\
M\end{array}$ & $\begin{array}{c}\text { M } \\
M \\
\text { F } \\
M \\
\text { F } \\
\text { M } \\
\text { F }\end{array}$ & $\begin{array}{c}\text { yes } \\
\text { yes } \\
\text { yes } \\
\text { no } \\
\text { yes } \\
\text { no } \\
\text { no }\end{array}$ & $\begin{array}{l}56 \\
35 \\
45 \\
21 \\
42 \\
42 \\
46 \\
41\end{array}$ & $\begin{array}{c}2 \\
3 \\
2 \\
5 \\
1 \\
0 \\
0 \\
\mathbf{1 , 8 6}\end{array}$ & $\begin{array}{c}1,2,3 \\
1,2,3 \\
1 \\
2,3 \\
1 \\
1,3 \\
3\end{array}$ \\
\hline ڤn & $\begin{array}{c}4 \\
6 \\
8 \\
11 \\
13 \\
17 \\
20 \\
M\end{array}$ & $\begin{array}{l}\mathrm{M} \\
\mathrm{M} \\
\mathrm{F} \\
\mathrm{M} \\
\mathrm{M} \\
\mathrm{F} \\
\mathrm{F}\end{array}$ & $\begin{array}{l}\text { yes } \\
\text { yes } \\
\text { yes } \\
\text { yes } \\
\text { yes } \\
\text { yes } \\
\text { yes }\end{array}$ & $\begin{array}{l}47 \\
57 \\
49 \\
36 \\
61 \\
41 \\
31 \\
46\end{array}$ & $\begin{array}{c}5 \\
2 \\
3 \\
6 \\
2 \\
4 \\
1 \\
\mathbf{3 , 2 9}\end{array}$ & $\begin{array}{c}\text { No experience } \\
1,2,3 \\
3 \\
1,2,3 \\
1,3 \\
1 \\
1\end{array}$ \\
\hline
\end{tabular}

Table 1. Participant description according to groups

\subsection{Materials}

\subsubsection{Questionnaire Design}

Participants were tested using an adapted version of the questionnaire outlined in the manual of London, Rother, Tolosana, and Maruping's (2003) Hazard Communication Comprehensibility Testing. This manual is based on the methodology used by the OEHRU at the University of Cape Town in a study to support the implementation of the GHS in South Africa. It was selected because of its emphasis on developing an instrument that is "globally applicable taking into account varying levels of literacy and differences in cultural experience" (London et al. 2003: 1). Questions were adapted from modules 2 and 3 of the manual and translated into Afrikaans. The final questionnaire consisted of three sections, as outlined in Table 2. 


\begin{tabular}{|l|l|l|}
\hline Section & \multicolumn{1}{|c|}{ Title } & \multicolumn{1}{c|}{ Aims } \\
\hline Section 1 & Pretest Participant Interview & $\begin{array}{l}\text { To ascertain specific data concerning demographics and work } \\
\text { experience that may influence interpretation of comprehensibility } \\
\text { results. }\end{array}$ \\
\hline Section 2 & $\begin{array}{l}\text { Testing of Hazard Sign } \\
\text { Comprehensibility }\end{array}$ & $\begin{array}{l}\text { To evaluate participants' familiarity with each sign. } \\
\text { To test the comprehensibility of each sign. } \\
\text { To determine how difficult each sign is to understand. } \\
\text { To identify suggestions that could make each sign easier to } \\
\text { understand. }\end{array}$ \\
\hline Section 3 & Post-test Participant Interview & $\begin{array}{l}\text { To determine suggestions regarding the type of safety information } \\
\text { that should appear on chemicals. } \\
\text { To determine suggestions regarding how this information should } \\
\text { be presented. }\end{array}$ \\
\hline
\end{tabular}

Table 2. Outline of the questionnaire (adapted from London et al. 2003)

\subsubsection{Picture Stimuli}

Eight hazard signs used by the GHS were selected for comprehensibility testing in the present study. These signs were selected because they frequently appear on commonly used chemicals. Six of these signs had also been used in previous studies to assess hazard sign comprehensibility (Wilkinson et al. 1997; GHS Study 2003). This was important to enable cross-study comparisons between results. The eight hazard signs appear in Figure 1.

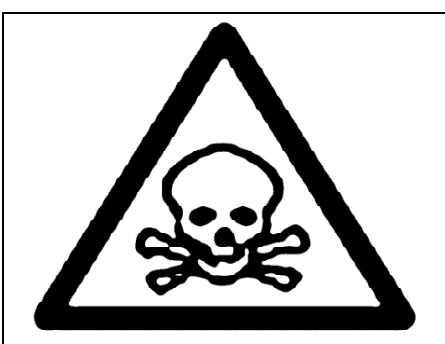

Skull \& Crossbones

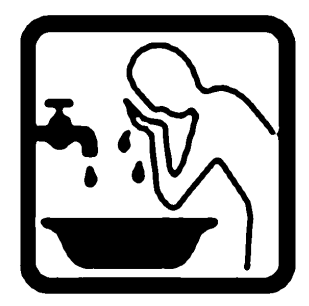

Wash Hands \& Face After Use

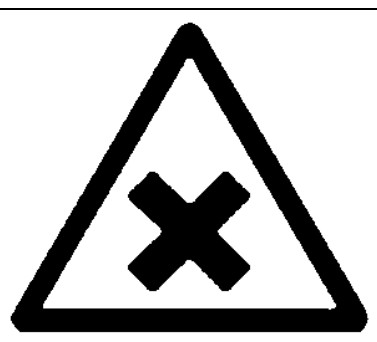

Harmful

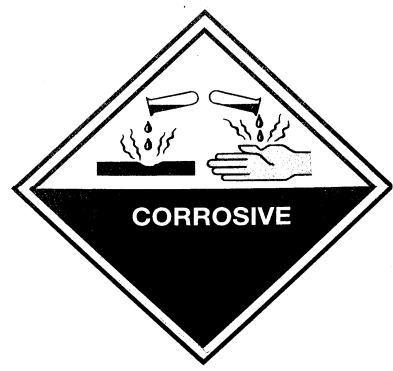

Corrosive

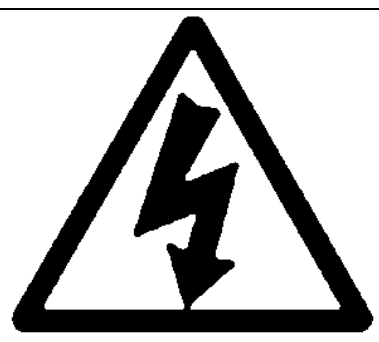

High Voltage

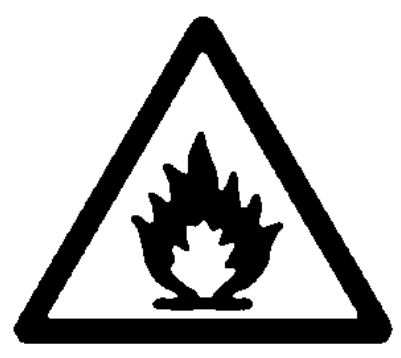

Flammable

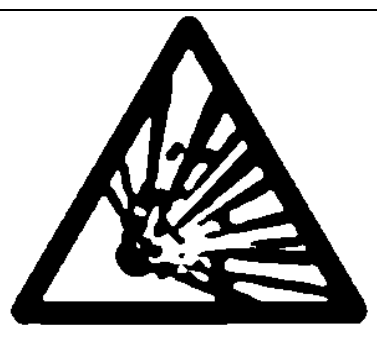

Explosive

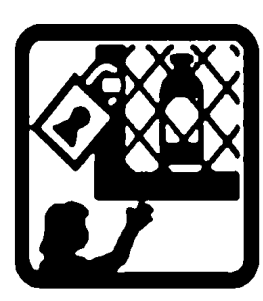

Keep Out of Reach of Children

Figure 1. The eight hazard signs tested 
Three different versions of each of these signs were developed for the purposes of the present research project:

1. The first version displayed each sign on its own, printed in black and white.

2. The second version provided each sign with a corresponding written translation, also in black and white, the rationale being that, by pairing signs with written warnings, the two may become associated in memory (Young and Wogalter 1990). This may then aid retrieval of hazard information when the sign is seen again (Wilkinson et al. 1997). These written translations were given in English, as this is the language in which they predominantly appear in natural contexts.

3. The third version provided the sign within a meaningful pictured context. Context is particularly important when assessing hazard sign comprehensibility, as contextual cues greatly contribute to comprehension (Johansen and Larsen 2002). This is particularly so for populations with little formal education (London et al. 2003).

\subsection{Research procedure}

The study was conducted by means of personal interviews with the first author as the interviewer. Once the study had been explained and consent obtained, participants were randomly divided into three groups of seven participants each. Each group was exposed to one of the three versions of hazard signs described above. The participants were individually guided through the questionnaire and presented with the hazard signs one at a time. The hazard signs were randomly ordered and were presented to all participants in the same order. The participants were allowed to view each sign for 10 seconds before being asked questions about it. They were permitted to retain the sign while answering these questions.

The participants' suggestions regarding how signs could be improved were obtained using open-ended questions and limited prompting where necessary. All responses were audio recorded for later classification. At the end of the interview each participant was rewarded with a safety information card containing relevant safety information for their workplace or home. 


\subsection{Data analysis}

Data analyses aimed at providing answers to the two main research questions. Answers to closed questions were coded into one of two categories: correct and incorrect. Participants who were unable to provide an answer were coded as incorrect. Only spontaneous correct responses were coded as correct.

Each hazard sign was assessed in terms of its comprehensibility and compared to the other hazard signs in its group. Answers to open-ended questions were transcribed and, where applicable, grouped into specific categories of suggestions. Percentages and means were used to make comparisons between data, the sample size being sufficient to allow this (Howell 2004).

\section{Results and discussion}

\subsection{Comprehensibility ratings}

Results of the comprehensibility measurements revealed a moderate understanding of the eight hazard signs used. Overall, $54.17 \%$ of the responses were coded as correct. The comprehensibility of individual signs varied greatly. The $>50 \%$ overall correct response could be largely ascribed to three signs that received over $80 \%$ correct responses, namely the "skulland-crossbones" (90.48\%), "wash hands and face after use" (90.48\%) and "flammable" signs $(80.95 \%)$ (cf. Table 3).

\begin{tabular}{|l|c|}
\hline \multicolumn{1}{|c|}{ Hazard Sign: } & \% Correct Responses \\
\hline Skull-\&-Crossbones & 90.48 \\
\hline Wash Hands and Face After Use & 90.48 \\
\hline Flammable & 80.95 \\
\hline Corrosive & 47.62 \\
\hline High Voltage & 42.86 \\
\hline Keep Out of Reach of Children & 38.10 \\
\hline Explosive & 23.81 \\
\hline Harmful & 19.05 \\
\hline $\begin{array}{l}\text { Total \% Correct Responses (all } \\
\text { signs) }\end{array}$ & $\mathbf{5 4 . 1 7}$ \\
\hline $\begin{array}{l}\text { Total \% Correct Responses } \\
\text { (top 3 signs omitted) }\end{array}$ & $\mathbf{3 4 . 2 9}$ \\
\hline
\end{tabular}

Table 3. Order and percentage correct responses for each sign

The percentage of correct responses for the five remaining signs stood at $34.29 \%$. Reasons for this inconsistency in the comprehensibility of the three most comprehensible signs and the 
remaining signs can possibly be explained by looking at what makes these three signs so much easier to comprehend.

Participants' previous exposure appeared to be important in the case of the "skull-andcrossbones" and "flammable" signs. Twenty and 17 participants, respectively, recognized these as signs they had seen before. This concurs with literature that has identified previous exposure to signs as an important determiner in their recall and comprehension (Johansen and Larsen 2002; GHS Study 2003). Previous exposure, however, had limited validity for the third sign, namely "wash hands and face after use". Only 13 out of the 21 participants indicated that they had seen it before. Factors other than previous exposure, e.g., iconicity, were likely to have played a role in the comprehensibility of this particular sign. The "wash hands and face after use" sign appeared to have a high degree of transparency for participants. This is suggested by the ease of perception ratings for this sign (see Figure 1c). It can be seen that $80.95 \%$ of the participants rated this sign as "easy to understand", and $90.48 \%$ correctly identified its meaning. The suggestion of iconicity as a contributing factor to the high percentage of comprehension for this particular sign is supported by the findings in the GHS Study (2003), where more than $90 \%$ of the participants correctly or partially correctly identified the "wash hands and face after use" sign. Literature abounds with information regarding the influence of iconicity on sign comprehension (Fuller and Lloyd 1997; Hollosi 2000; Johansen and Larsen 2002; Beukelman and Mirenda 2005).

Comparison of results in figure 1 also revealed some contradictions between the comprehensibility scores and previous exposure scores of the "high voltage" and "harmful" hazard signs. The "high voltage" sign ranked as the fourth most understood sign, with $42.86 \%$ correct responses. Its previous exposure rating, however, revealed it to be the second most recognized sign out of the eight tested. It was also perceived as one of the top three most easily understood signs. A similar pattern was observed for the "harmful" sign. This sign received the least correct comprehension responses (19.05\%), but had the second highest previous exposure rating and was the third most easily perceived sign. 


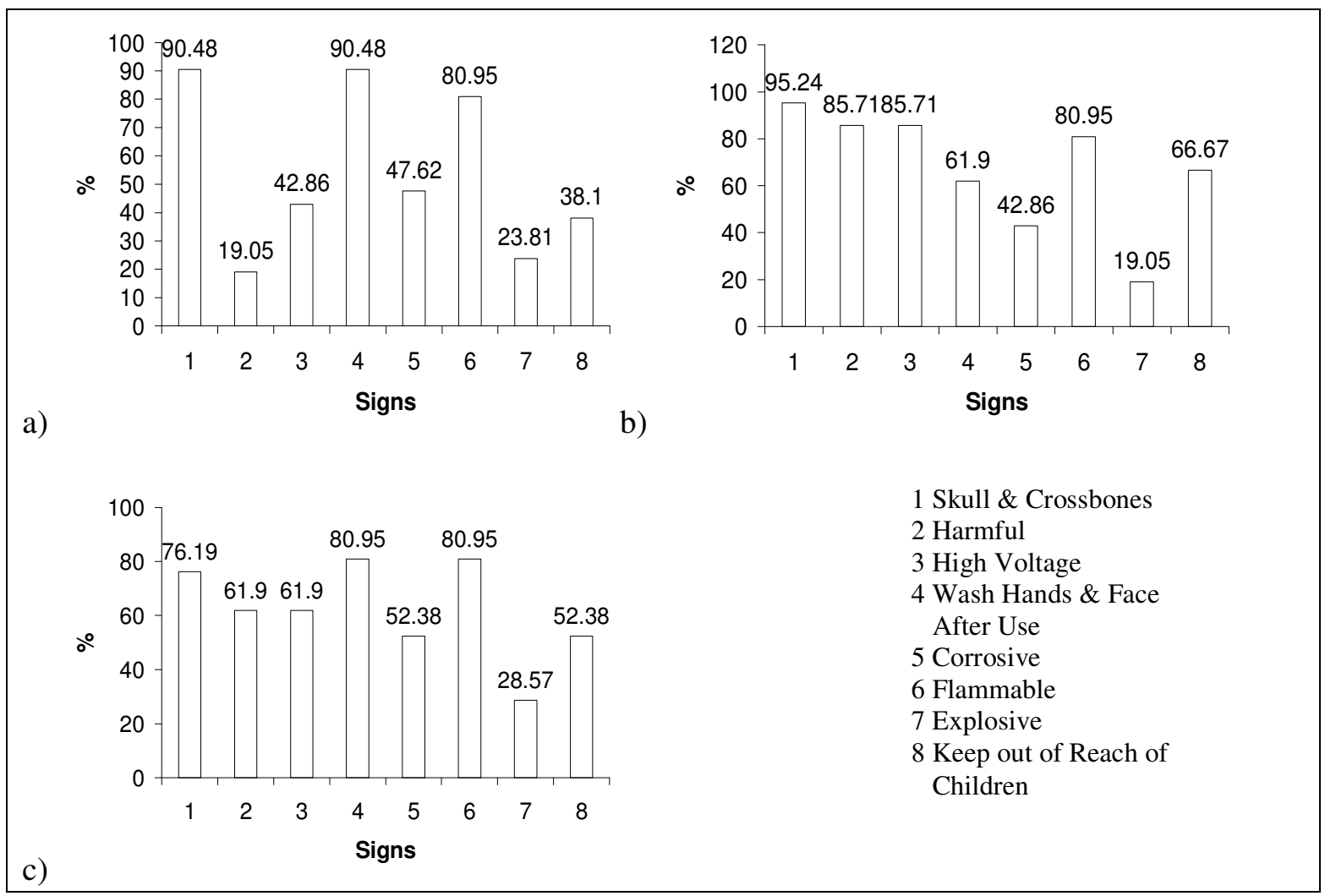

Figure 2. Percentages for (a) comprehensibility, (b) previous exposure, and (c) ease of perception

Reasons for these contradictions can possibly be ascribed to confusions between these signs and other similar common signs. The "high voltage" sign, for example, was misidentified as the road sign indicating a sharp turn by five of the 21 participants. Other misinterpretations were that the lightening bolt indicated an arrow where emergency help could be obtained, or where there was a high risk of accidents.

Confusion was even more clearly evident for the "harmful" sign, and explains the contrasting results between comprehensibility, previous exposure, and ease of perception. No less than eight different interpretations of this sign were recorded. The two most common of these were the hospital/ambulance cross (five participants) and the railway line (three participants). Other misinterpretations involved the "no entry" sign, the "incorrect/mistake" sign, the "danger of fire" sign, and warning signs of any kind. Almost all the participants (95\%) identified it as a warning sign. One participant thought it meant the opposite of its intended message. The generalization of meanings to other signs is not uncommon, and may enhance the training of hazard sign comprehension by using more easily comprehended signs to improve intelligibility of other signs (GHS Study 2003). 


\subsection{Comparison between different types of sign presentation}

Comparison of the different types of sign presentation revealed the expected results. Participants in group 1, who were shown the signs alone, yielded the fewest correct responses $(42.86 \%)$. They were followed by those in group 2 , who were shown the signs with written translations. The comprehensibility percentage for this group was 55.36\%. Participants in group 3, who were shown the signs within a meaningful context, obtained the highest percentage $(64.24 \%)$ of correct responses.

These results have important implications for hazard sign design and use, particularly among illiterate populations. Firstly, a sign presented alone is clearly insufficient in communicating a comprehensive warning message. Although previous exposure to these signs may help in recognition, it does not significantly aid comprehension.

The second implication suggests that the addition of written text to hazard signs seems to increase comprehensibility, even for illiterate adults, and even when not written in their mother tongue. There was a $12.5 \%$ increase in comprehensibility between groups 1 and 2 . This may be ascribed to two reasons, namely the addition of the written text to signs and/or inter-group variation. Since group differences were controlled to some degree by random participant assignment (Bless and Higson-Smith 2004), and all other variables were kept constant, this $12.5 \%$ increase may well have been due to the presence of the written text in the case of group 2. The exact reason as to why the addition of written text should increase sign comprehension for illiterate adults is not clear and requires further investigation. It is possible that the participants were not as illiterate as was assumed.

The last implication is that the inclusion of a meaningful context enhances hazard sign comprehensibility. From the results in this study it was clear that the context in which a sign appeared played a significant role in improving access to its meaning. There was a $21.43 \%$ increase in comprehensibility between groups 1 and 3, and an $8.93 \%$ increase between groups 2 and 3, respectively. These increases can be ascribed to one of two factors, namely intergroup variation and the inclusion of a meaningful context. It is logical to assume that these increases were, at least to a degree, the result of the contexts in which the signs appeared. This result is comforting, as in most practical cases the hazard signs occur in context, which clearly helps their interpretation. 


\subsection{Suggestions for improvements in sign design}

The third and final aim of this study was to elicit suggestions for how to improve hazard sign comprehensibility. These suggestions fell into seven categories: colour, writing, size, translation, training, clarity, and additional pictures/signs.

For groups 1 and 2, adding colour was rated high. It is interesting to note that both group 1 and group 2 participants were presented with black and white signs. Group 1 participants also suggested adding writing and increasing size to improve comprehensibility, while participants in group 2 suggested more training and improved clarity. Thus, although simple black and white line drawings can be regarded as the simplest form of presentation, the present findings suggest that colour simplicity does not aid comprehension. The inclusion of colours, particularly red, may be a key element in the improvement of hazard sign comprehension. For

participants in group 3, clarity, size, training, and the addition of a picture or another sign were the most suggested modifications. This group gave the fewest suggestions of the three.

\subsection{Implications of results}

The findings of this study suggest that poor hazard sign comprehension may be a reason for some of the poison and burn victims seen daily in South African hospitals. The overall poor comprehensibility of hazard signs indicates a need for collaborative partnerships between communication experts, such as Speech-Language Therapists, hazard sign designers and producers of hazardous substances alike to improve safety communication. By encouraging these partnerships, signs with improved transparency and form of presentation can be developed which, in turn, could promote easier access to hazard and safety information, particularly among illiterate populations.

These partnerships could also assist in the establishment of standardized signs for specific hazards. This is of particular importance in South Africa, where no standardized warning system to which all sectors are forced to adhere currently exists (GHS Study 2003). It is also useful to highlight a potential obstacle to the suggested improvements. The decanting of hazardous substances, such as paraffin and petrol, into unmarked containers, is common practice in South Africa, particularly in poorer communities (GHS Study 2003). Since these are also the communities most affected by the misuse and abuse of hazardous substances, something needs to be done to ensure that safety information is communicated, despite this 
decanting practice (e.g. education of "spaza" shop or café owners regarding relevant safety measures). Adhesive hazard sign stickers along with educational pamphlets might also be distributed to these shop owners, so that all bottles used for decanting can be clearly marked.

Another implication of the present study is the need for training. The low levels of hazard sign comprehensibility found in this study indicate the need for widespread training programmes that target not only hazard sign comprehension, but also sign recall and recognition. Reinforcing this conclusion is the fact that training was rated in the top four suggestions for hazard sign improvement across the three groups in the results above. This indicates that hazard sign users also perceive training as an important aspect of increasing hazard sign comprehensibility. The responsibility for these training programs lies largely with those who manufacture, distribute and use hazardous substances. Employers in South Africa are required by law to ensure the safety of all individuals working for them and therefore should be the primary providers of these programmes (Occupational Health and Safety Act 1993). Since communication, by definition, incorporates the need for the successful transfer of information, speech-language therapists could play a key role in the development of training programmes that effectively convey hazard sign information.

\subsection{Strengths and weaknesses of the study}

Only 21 participants participated in the study, affecting the generalizability of the findings to the larger South African population. A repeat of this study making use of a larger test population may or may not provide support for the results reported here. Another weakness of the present study is the fact that participants were selected from only one linguistic group. This has provided very specific results that might only be applicable to Afrikaans speaking populations.

\subsection{Suggestions for future studies}

Although this study contributes to the growing body of research on hazard sign comprehensibility, much is still unknown about how to make hazard information more accessible through signs. It would be valuable to determine whether literacy level does, in fact, affect hazard sign comprehension, and, if so, to determine the minimum level of literacy or education needed to facilitate sign comprehension. The role of compliance to hazard signs, in addition to their comprehension, should also be researched, as it is not only necessary to 
understand what is needed to avoid harm, but also to carry out the necessary actions. Other questions requiring answers may be: At which point do signs become truly oblique for illiterate adults, and in which contexts are signs most easily understood? Within a similar research design, different cultures, age groups, and literate populations could also be tested in order to further establish the scope of hazard sign incomprehensibility in South Africa.

\section{Conclusion}

The results of the present study add to the growing body of research on the comprehensibility of hazard signs. Although the comprehensibility ratings indicated an average understanding of the eight hazard signs, the score appeared "artificially" elevated due to three signs which were much better understood than the others. Comprehensibility results from the different types of sign presentation indicated that participants who were presented with signs in context showed the highest levels of comprehension. Interestingly, signs presented with their corresponding written texts received elevated scores, even though the participants were considered illiterate.

Speech-language therapists, as communication specialists, could have a valuable contribution to make to comprehensibility training, further research, and hazard sign iconicity and design. Consideration of the suggestions made by sign users themselves is essential for improving sign appeal and comprehension. Although some practical suggestions were given, further research is clearly needed.

\section{Acknowledgements}

The authors would like to acknowledge the support of Daleen Klop, Head of the Department of Speech-Language and Hearing Therapy at the University of Stellenbosch, for her helpful resources, and the staff of the Matie Community Service Adult Basic Education and Training program, particularly Ms Linda Jacobs. The work that is done in this programme is commendable beyond words. 


\section{References}

Andrews, E. 2000. The role of semiotics in the study of language, linguistics and communication: an overview. http://www.indiana.edu/ slavconf/SLING2K/pospapers/ andrews.pdf. Accessed on 20 October 2005.

Basson, M. and E. Alant. 2005. The iconicity and ease of learning of picture communication symbols: A study with Afrikaans-speaking children. The South African Journal of Communication Disorders 25: 4-11.

Barbarin, O.A. and L.M. Richter. 2001. Mandela's children: Growing up in post-apartheid South Africa. New York: Routledge.

Beukelman, D. and P. Mirenda. 2005. Augmentative and alternative communication for children and adults with communicative needs. $3^{\text {rd }}$ edition. Baltimore: Paul $\mathrm{H}$. Brookes.

Bless, C. and C. Higson-Smith. 2004. Social research methods: An African perspective. $3^{\text {rd }}$ edition. Cape Town: Juta Education.

Blischak, D.M., L.L. Lloyd, and D.R. Fuller. 1997. Terminology issues. In L.L. Lloyd, D.R. Fuller, and H.H. Arvidson (Eds). Augmentative and alternative communication. A handbook of principles and practices. Boston: Allyn and Bacon. pp. 38-42.

Chandler, D. 1994. Semiotics for beginners. http://www.aber.ac.uk/media/Documents/S4B/ Accessed on 14 January 2006.

Davies, S., H.M. Haines, and B.J. Norris. 2000. The role of pictograms in the conveying of consumer safety information. http://www.dti.gov.uk/homesafetynetwork/pdf/picto.pdf. Accessed on 24 October 2005.

Department of Trade and Industry. 2001. Poisonings - household chemicals. http://www.dti.gov.uk/homesafetynetwork/cp_stats.htm. Accessed on 15 January 2006.

Department of Trade and Industry. 1999. Development of further warning symbols for packaging. http://www.dti.gov.uk/homesafetynetwork/pdf/warning.pdf. Accessed on 24 October 2005.

Dunham, J.K. 1989. The transparency of manual signs in a linguistic and an environmental nonlinguistic context. Augmentative and Alternative Communication 11: 214-225.

Fristoe, M., and L.L. Lloyd. 1979. Nonspeech communication. In N.R. Ellis (ed). Handbook of mental deficiency: Psychological theory and research $2^{\text {nd }}$ ed. New York: Lawrence Erlbaum Associates. pp. 401-430. 
Fuller, D.R., and L.L. Lloyd. 1997. Symbol selection. In L.L. Lloyd, D.R. Fuller, and H.H. Arvidson (eds). Augmentative and alternative communication. A handbook of principles and practices. Boston: Allyn and Bacon. pp. 214-255.

Fuller, D.R., and M.M. Stratton. 1991. Representativeness verses translucency: Differential theoretical backgrounds, but are they different concepts? A position paper. Augmentative and Alternative Communication 7: 51-58.

Global Harmonized System for the Classification and Labeling of Chemicals (GHS) Study Part 1: Situation Analysis. 2003. Executive Summary. http:www.nedlac.org.za/ research. Accessed on 3 February 2006.

Hanna, A., and R. Remington. 1996. The representation of colour and form in long-term memory. Memory and Cognition 24: 322-320.

Hollosi, A. 2000. Alternative representations and beyond: A new proposal for a multi-sensory language interface (MUSLI). http://www.iicm.edu/thesis/ahollosi_html/node8.html. Accessed on 20 October 2005.

Howell, D.C. 2004. Fundamental statistics for the behavioural sciences $5^{\text {th }}$ ed. Belmont: Thomson Brookes/Cole.

Huer, M.B. 2000. Examining perceptions of graphic symbols across cultures: preliminary study of the impact of culture/ethnicity. Augmentative and Alternative Communication 16: $180-185$.

Johansen, J.D., and S.E. Larsen. 2002. Signs in use: an introduction to semiotics. New York: Routledge.

Kalaroa, N. 2005. Breaching the language barrier. http:// www.ohsonline.com/Stevens/ OHSPub.nsf/Articles2/E3E900953CF0615F86256EA1006D11E1?OpenDocument. Accessed on 24 October 2005.

Kristeva, J. 1989. Language the unknown: An introduction into linguistics. New York: Columbia University Press.

Lesch, M.F. 2005. Signs of age in today's workforce. http://www.ohsonline.com/Stevens/ OHSPub.nsf/Articles2/B6FAC60CEA76D04686256C79006F7A0B?OpenDocument. Accessed on 24 October 2005.

London, L., A. Rother, S. Tolosana, and M. Maruping. 2003. Hazard communication comprehensibility testing - tool and toolkit. http://www.nedlac.org.za/top.asp?inc= research /fridge/ghs1203part2/index.htm. Accessed on 10 February 2006.

Mizuko, M. 2004. Communication Symbols. http://www.d.umn.edu/ mmizuko/5230/symbols. 
htm. Accessed on 20 October 2005.

Occupational and Environmental Health Research Unit. 2003. GHS study-chemical hazard communication comprehensibility testing. http://www.nedlac.org.za/research/fridge/ghs1203_ part3/chapt7.pdf. Accessed on 24 October 2005.

S.A. Department of Labour. Occupational Health and Safety Act No. 85. 1993. http://www. labourguide.co.za/Occupational\%20Health\%20and\%20Safety.doc. Accessed on 20 June 2006.

Soto, G., and W. Olmstead. 1993. A semiotic perspective for AAC. Augmentative and Alternative Communication 9: 134-141.

South Africa. Ministry for Welfare and Population Development. 1997. White paper for social welfare: Principles, guidelines, recommendations, proposed policies and programs for developmental social welfare in South Africa. http:// www.polity. org.za/ govdocs/white_paper/social971.html. Accessed on 10 February 2006.

Stephenson, J., and K. Linfoot. 1996. Pictures as communication symbols for students with severe intellectual disability. Augmentative and Alternative Communication 12(4): 244-255.

Terumasa, K., and H. Takeshi. 1999. Hemisphere specialization and categorical spatial relations representations. Laterality 4: 321-331.

Tsal, Y., and T. Bareket. 1999. Effects of attention on localization of stimuli in the visual field. Psychonomic Bulletin and Review 6: 292-296.

Underwood, P.G. 2002. South Africa: A case study in development through information literacy. White Paper prepared for UNESCO, the U.S. National Commission on Libraries and Information Science, and the National Forum on Information Literacy. http://www.nclis.gov/libinter/infolitconf\&meet/papers/underwood-fullpaper.pdf. Accessed on 26 October 2005.

United Nations Development Program. 2002. Human development report 2001. http://www.undp.org/hdr2001/indicator/cty_f_ZAF.html. Accessed on 10 February 2006.

Van Heerden, G. 1991. Meeting Needs. NU Focus 2(3): 4.

Wilkinson, R.L., J.W. Cary, N.F. Barr, and J. Reynolds. 1997. Comprehension of pesticide safety information: Effects of pictorial and textual warnings. International Journal of Pest Management 43(3): 239-245. 
Wilkinson, K.M., and V. Jagaroo. 2004. Contributions of principles of visual cognitive science to AAC system display design. Augmentative and Alternative Communication 20(3): 123-136.

Workforce Investment Act. 1998. http://www.vdoe.vi.virginia/gov/instruction/adulted/ resources/forms/wia_titles.pdf. Accessed on 20 June 2006.

Young, S.L., and M.S. Wogalter. 1990. Comprehension and memory of instruction manual warnings: Conspicuous print and pictorial icons. Human Factors 32: 637-649. 